\title{
New Outer Bounds on the Capacity Region of Gaussian Interference Channels
}

\author{
Xiaohu Shang \\ Syracuse University \\ Department of EECS \\ Email: xshang@syr.edu
}

\author{
Gerhard Kramer \\ Bell Labs \\ Alcatel-Lucent \\ Email: gkr@ research.bell-labs.com
}

\author{
Biao Chen \\ Syracuse University \\ Department of EECS \\ Email: bichen@ecs.syr.edu
}

\begin{abstract}
Recent outer bounds on the capacity region of Gaussian interference channels are generalized to $m$-user channels with $m>2$ and asymmetric powers and crosstalk coefficients. The bounds are again shown to give the sum-rate capacity for Gaussian interference channels with low powers and crosstalk coefficients. The capacity is achieved by using single-user detection at each receiver, i.e., treating the interference as noise incurs no loss in performance.
\end{abstract}

Index terms - capacity, Gaussian noise, interference.

\section{INTRODUCTION}

This paper extends the results of [1] to asymmetric Gaussian ICs. The paper further has a new Theorem (Theorem 4) that is not in [2] or in other recent works (see Section V and Motahari and Khandani [3], and Annapureddy and Veeravalli [4]).

The interference channel (IC) models communication systems where transmitters communicate with their respective receivers while causing interference to all other receivers. For a two-user Gaussian IC, the channel output can be written in the standard form [5]

$$
\begin{aligned}
& Y_{1}=X_{1}+\sqrt{a} X_{2}+Z_{1}, \\
& Y_{2}=\sqrt{b} X_{1}+X_{2}+Z_{2},
\end{aligned}
$$

where $\sqrt{a}$ and $\sqrt{b}$ are channel coefficients, $X_{i}$ and $Y_{i}$ are the transmit and receive signals. The user/channel input sequence $X_{i 1}, X_{i 2}, \cdots, X_{i n}$ is subject to the power constraint $\sum_{j=1}^{n} \mathcal{E}\left(X_{i j}^{2}\right) \leq n P_{i}, i=1,2$. The transmitted signals $X_{1}$ and $X_{2}$ are statistically independent. The channel noises $Z_{1}$ and $Z_{2}$ are possibly correlated unit variance Gaussian random variables, and $\left(Z_{1}, Z_{2}\right)$ is statistically independent of $\left(X_{1}, X_{2}\right)$. In the following, we denote this Gaussian IC as $\operatorname{IC}\left(a, b, P_{1}, P_{2}\right)$.

The capacity region of an IC is defined as the closure of the set of rate pairs $\left(R_{1}, R_{2}\right)$ for which both receivers can decode their own messages with arbitrarily small positive error probability. The capacity region of a Gaussian IC is known only for three cases: (1) $a=0, b=0$. (2) $a \geq 1, b \geq 1$ : see [6]-[8]. (3) $a=0, b \geq 1$; or $a \geq 1, b=0$ : see [9]. For the second case both receivers can decode the messages of both transmitters. Thus this IC acts as two multiple access channels (MACs), and the capacity region for the IC is the intersection of the capacity region of the two MACs. However, when the interference is weak or moderate, the capacity region is still unknown. The best inner bound is obtained in [8] by using superposition coding and joint decoding. A simplified form of the Han-Kobayashi region was given by Chong-MotaniGarg-El Gamal [10], [11]. Various outer bounds have been developed in [12]-[16]. Kramer in [14] presented two outer bounds. The first is obtained by providing each receiver with just enough information to decode both messages. The second is obtained by reducing the IC to a degraded broadcast channel. Both bounds dominate the bounds by Sato [12] and Carleial [13]. The recent outer bounds by Etkin, Tse, and Wang in [15] are also based on genie-aided methods, and they show that Han and Kobayashi's inner bound is within one bit or a factor of two of the capacity region. This result can also be established by the methods of Telatar and Tse [16]. We remark that neither of the bounds of [14] and [15] implies each other. Numerical results show that the bounds of [14] are better at low SNR while those of [15] are better at high SNR. The bounds of [16] are not amenable to numerical evaluation since the optimal distributions of the auxiliary random variables are unknown.

In this paper, we present new outer bounds on the capacity region of Gaussian ICs that generalize results of [1]. The new bound is based on a genie-aided approach and an extremal inequality proposed by Liu and Viswanath [17]. Based on this outer bound, we obtain new sum-rate capacity results for ICs satisfying some channel coefficient and power constraint conditions. We show that the sum-rate capacity can be achieved by treating the interference as noise when both the channel gain and the power constraint are weak. We say that such channels have noisy interference. For this class of interference, the simple single-user transmission and detection strategy is sum-rate optimal.

This paper is organized as follows. In Section II, we present an outer bound and the resulting sum-rate capacity for certain 2-user Gaussian ICs. An extension of the sum-rate capacity under noisy interference to $m$-user ICs is provided in Section III. Numerical examples are given in Section IV, and Section $\mathrm{V}$ concludes the paper.

\section{A Genie-AIDED Outer Bound}

\section{A. General outer bound}

The following is a new outer bound on the capacity region of Gaussian ICs. Note that in contrast to [1] these bounds permit $P_{1} \neq P_{2}$ and $a \neq b$. 


$$
\begin{aligned}
R_{1}+\mu R_{2} \leq & \min _{\substack{\rho_{i} \in[0,1] \\
\left(\sigma_{1}^{2}, \sigma_{2}^{2}\right) \in \Sigma}} \frac{1}{2} \log \left(1+\frac{P_{1}^{*}}{\sigma_{1}^{2}}\right)-\frac{1}{2} \log \left(a P_{2}^{*}+1-\rho_{1}^{2}\right)+\frac{1}{2} \log \left(1+P_{1}+a P_{2}-\frac{\left(P_{1}+\rho_{1} \sigma_{1}\right)^{2}}{P_{1}+\sigma_{1}^{2}}\right) \\
& \quad+\frac{\mu}{2} \log \left(1+\frac{P_{2}^{*}}{\sigma_{2}^{2}}\right)-\frac{\mu}{2} \log \left(b P_{1}^{*}+1-\rho_{2}^{2}\right)+\frac{\mu}{2} \log \left(1+P_{2}+b P_{1}-\frac{\left(P_{2}+\rho_{2} \sigma_{2}\right)^{2}}{P_{2}+\sigma_{2}^{2}}\right) \\
R_{1}+\eta_{1} R_{2} \leq & \frac{1}{2} \log \left(1+\frac{b \eta_{1}-1}{b-b \eta_{1}}\right)-\frac{\eta_{1}}{2} \log \left(1+\frac{b \eta_{1}-1}{1-\eta_{1}}\right)+\frac{\eta_{1}}{2} \log \left(1+b P_{1}+P_{2}\right) \\
R_{1}+\eta_{2} R_{2} \leq & \frac{1}{2} \log \left(1+P_{1}+a P_{2}\right)-\frac{1}{2} \log \left(1+\frac{a-\eta_{2}}{\eta_{2}-1}\right)+\frac{\eta_{2}}{2} \log \left(1+\frac{a-\eta_{2}}{a \eta_{2}-a}\right) .
\end{aligned}
$$

Theorem 1: If the rates $\left(R_{1}, R_{2}\right)$ are achievable for $\operatorname{IC}\left(a, b, P_{1}, P_{2}\right)$ with $0<a<1,0<b<1$, they must satisfy the following constraints (1)-(3) for $\mu>0, \frac{1+b P_{1}}{b+b P_{1}} \leq \eta_{1} \leq \frac{1}{b}$ and $a \leq \eta_{2} \leq \frac{a+a P_{2}}{1+a P_{2}}$, where

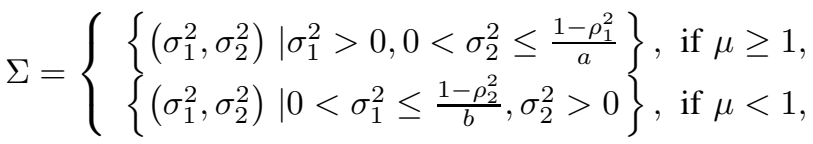

and if $\mu \geq 1$ we define

$$
\begin{aligned}
& P_{1}^{*}= \begin{cases}P_{1}, & \sigma_{1}^{2} \leq\left[\frac{(1-\mu) P_{1}}{\mu}+\frac{1-\rho_{2}^{2}}{b \mu}\right]^{+}, \\
\frac{1-\rho_{2}^{2}-b \mu \sigma_{1}^{2}}{b \mu-b}, & {\left[\frac{(1-\mu) P_{1}}{\mu}+\frac{1-\rho_{2}^{2}}{b \mu}\right]^{+}<\sigma_{1}^{2} \leq \frac{1-\rho_{2}^{2}}{b \mu},(5)} \\
0, & \sigma_{1}^{2}>\frac{1-\rho_{2}^{2}}{b \mu},\end{cases} \\
& P_{2}^{*}=P_{2}, \\
& \sigma_{2}^{2} \leq \frac{1-\rho_{1}^{2}}{a},
\end{aligned}
$$

where $(x)^{+} \triangleq \max \{x, 0\}$, and if $0<\mu<1$ we define

$$
\begin{aligned}
& P_{1}^{*}=P_{1}, \\
& P_{2}^{*}= \begin{cases}P_{2}, & \sigma_{1}^{2} \leq \frac{1-\rho_{2}^{2}}{b}, \\
\frac{\mu\left(1-\rho_{1}^{2}\right)-a \sigma_{2}^{2}}{a-a \mu}, & \left.\left[(\mu-1) P_{2}+\frac{\mu\left(1-\rho_{1}^{2}\right)}{a}\right]^{+}\right]^{+}, \\
0, & \sigma_{2}^{2}>\frac{\mu\left(1-\rho_{1}^{2}\right)}{a} .\end{cases}
\end{aligned}
$$

Proof: We give a sketch of the proof. A genie provides the two receivers with side information $X_{1}+N_{1}$ and $X_{2}+N_{2}$ respectively, where $N_{i}$ is Gaussian distributed with variance $\sigma_{i}^{2}$ and $\mathcal{E}\left(N_{i} Z_{i}\right)=\rho_{i} \sigma_{i}, i=1,2$. Starting from Fano's inequality, we have

$$
\begin{aligned}
n & \left(R_{1}+\mu R_{2}\right)-n \epsilon \\
\leq & I\left(X_{1}^{n} ; Y_{1}^{n}\right)+\mu I\left(X_{2}^{n} ; Y_{2}^{n}\right) \\
\leq & I\left(X_{1}^{n} ; Y_{1}^{n}, X_{1}^{n}+N_{1}^{n}\right)+\mu I\left(X_{2}^{n} ; Y_{2}^{n}, X_{2}^{n}+N_{2}^{n}\right) \\
= & {\left[h\left(X_{1}^{n}+N_{1}^{n}\right)-\mu h\left(\sqrt{b} X_{1}^{n}+Z_{2}^{n} \mid N_{2}^{n}\right)\right]-h\left(N_{1}^{n}\right) } \\
& +\left[\mu h\left(X_{2}^{n}+N_{2}^{n}\right)-h\left(\sqrt{a} X_{2}^{n}+Z_{1}^{n} \mid N_{1}^{n}\right)\right]-\mu h\left(N_{2}^{n}\right) \\
& +h\left(Y_{1}^{n} \mid X_{1}^{n}+N_{1}^{n}\right)+\mu h\left(Y_{2}^{n} \mid X_{2}^{n}+N_{2}^{n}\right),
\end{aligned}
$$

where $\epsilon \rightarrow 0$ as $n \rightarrow \infty$. For $h\left(Y_{1}^{n} \mid X_{1}^{n}+N_{1}^{n}\right)$, zero-mean Gaussian $X_{1}^{n}$ and $X_{2}^{n}$ with covariance matrices $P_{1} \mathbf{I}$ and $P_{2} \mathbf{I}$ are optimal, and we have

$$
\begin{aligned}
& h\left(Y_{1}^{n} \mid X_{1}^{n}+N_{1}^{n}\right) \\
& \leq \frac{n}{2} \log \left[2 \pi e\left(1+a P_{2}+P_{1}-\frac{\left(P_{1}+\rho_{1} \sigma_{1}\right)^{2}}{P_{1}+\sigma_{1}^{2}}\right)\right] .
\end{aligned}
$$

From the extremal inequality introduced in $[17$, Theorem 1 , Corollary 4], we have

$$
\begin{aligned}
& h\left(X_{1}^{n}+N_{1}^{n}\right)-\mu h\left(\sqrt{b} X_{1}^{n}+Z_{2}^{n} \mid N_{2}^{n}\right) \\
& \leq \frac{n}{2} \log \left[2 \pi e\left(P_{1}^{*}+\sigma_{1}^{2}\right)\right]-\frac{n \mu}{2} \log \left[2 \pi e\left(b P_{1}^{*}+1-\rho_{2}^{2}\right)\right],
\end{aligned}
$$

and

$$
\begin{aligned}
& \mu h\left(X_{2}^{n}+N_{2}^{n}\right)-h\left(\sqrt{a} X_{2}^{n}+Z_{1}^{n} \mid N_{1}^{n}\right) \\
& \leq \frac{n \mu}{2} \log \left[2 \pi e\left(P_{2}^{*}+\sigma_{2}^{2}\right)\right]-\frac{\mu}{2} \log \left[2 \pi e\left(a P_{2}^{*}+1-\rho_{1}^{2}\right)\right],
\end{aligned}
$$

where equalities hold when $X_{1}^{n}$ and $X_{2}^{n}$ are both Gaussian with covariance matrices $P_{1}^{*} \mathbf{I}$ and $P_{2}^{*} \mathbf{I}$ respectively. From (9)(12) we obtain the outer bound (11).

The outer bound in (2) (resp. (3)) is obtained by letting the genie provide side information $X_{2}$ to receiver one (resp. $X_{1}$ to receiver two), and applying the extremely inequality, i.e.,

$$
\begin{aligned}
n & \left(R_{1}+\eta_{1} R_{2}\right)-n \epsilon \\
\leq & I\left(X_{1}^{n} ; Y_{1}^{n}, X_{2}^{n}\right)+\eta_{1} I\left(X_{2}^{n} ; Y_{2}^{n}\right) \\
= & h\left(X_{1}^{n}+Z_{1}^{n}\right)-\eta_{1} h\left(\sqrt{b} X_{1}^{n}+Z_{2}^{n}\right)-h\left(Z_{1}^{n}\right) \\
& +\eta_{1} h\left(Y_{2}^{n}\right) \\
\leq & \frac{n}{2} \log \left(\tilde{P}_{1}+1\right)-\frac{n \eta_{1}}{2} \log \left(b \tilde{P}_{1}+1\right) \\
& +\frac{n \eta_{1}}{2} \log \left(1+b P_{1}+P_{2}\right),
\end{aligned}
$$

where $\tilde{P}_{1}=\frac{b \eta_{1}-1}{b-b \eta_{1}}$ for $\frac{1+b P_{1}}{b+b P_{1}} \leq \eta_{1} \leq \frac{1}{b}$. This is the bound in (2). Similarly, we obtain bound (3).

Remark 1: The bounds (1)-(3) are obtained by providing different genie-aided signals to the receivers. There is overlap of the range of $\mu, \eta_{1}$, and $\eta_{2}$, and none of the bounds uniformly dominates the other two bounds.

Remark 2: Equations (2) and (3) are outer bounds for the capacity region of a Z-IC, and a Z-IC is equivalent to a degraded IC [9]. For such channels, it can be shown that (9)(2) and (3) are the same as the outer bounds in [18]. For 
$\eta_{1}=\frac{1+b P_{1}}{b+b P_{1}}$ and $\eta_{2}=\frac{a+a P_{2}}{1+a P_{2}}$, the bounds in (2) and (3) are tight for a Z-IC (or degraded IC) because $\tilde{P}_{1}=P_{1}, \tilde{P}_{2}=P_{2}$ in (13), and there is no power sharing between the transmitters. Consequently, $\frac{1+b P_{1}}{b+b P_{1}}$ and $\frac{a+a P_{2}}{1+a P_{2}}$ are the negative slopes of the tangent lines for the capacity region at the corner points.

Remark 3: The bounds in (2)-(3) turn out to be the same as the bounds in [14, Theorem 2]. This can be shown by rewriting the bounds in [14, Theorem 2] in the form of a weighted sum rate.

Remark 4: The bounds in [14, Theorem 2] are obtained by getting rid of one of the interference links to reduce the IC into a Z-IC. In addition, the proof in [14] allowed the transmitters to share their power, which further reduces the Z-IC into a degraded broadcast channel. Then the capacity region of this degraded broadcast channel is an outer bound for the capacity region of the original IC. The bounds in (2) and (3) are also obtained by reducing the IC to a Z-IC. Although we do not explicitly allow the transmitters to share their power, it is interesting that these bounds are equivalent to the bounds in [14, Theorem 2] with power sharing. In fact, a careful examination of our derivation reveals that power sharing is implicitly assumed. For example, for the term $h\left(X_{1}^{n}+Z_{1}^{n}\right)-\eta_{1} h\left(\sqrt{b} X_{1}^{n}+Z_{2}^{n}\right)$ of (13), user 1 uses power $\tilde{P}_{1}=\frac{b \eta_{1}-1}{b-b \eta_{1}} \leq P_{1}$, while for the term $\eta_{1} h\left(Y_{2}^{n}\right)$ user 1 uses all the power $P_{1}$. This is equivalent to letting user 1 use the power $\tilde{P}_{1}$ for both terms, and letting user 2 use a power that exceeds $P_{2}$.

Remark 5: Theorem 1 improves [15, Theorem 3]. Specifically, the bound in (2) is tighter than the first sum-rate bound of [15, Theorem 3]. Similarly, the bound in (3) is tighter than the second sum-rate bound of [15, Theorem 3]. The third sumrate bound in [15, Theorem 3] is a special case of (1).

Remark 6: Our outer bound is not always tighter than that of [15] for all rate points. The reason is that in [15, last two equations of (39)], different genie-aided signals are provided to the same receiver. Our outer bound can also be improved in a similar and more general way by providing different genieaided signals to the receivers. Specifically the starting point of the bound can be modified to be

$$
\begin{aligned}
n\left(R_{1}+\mu R_{2}\right) \leq & \sum_{i=1}^{k} \lambda_{i} I\left(X_{1}^{n} ; Y_{1}^{n}, U_{i}\right) \\
& +\sum_{j=1}^{m} \mu_{i} I\left(X_{2}^{n} ; Y_{2}^{n}, W_{j}\right)+n \epsilon,
\end{aligned}
$$

where $\sum_{i=1}^{k} \lambda_{i}=1, \sum_{j=1}^{m} \mu_{j}=\mu, \lambda_{i}>0, \mu_{j}>0$.

\section{B. Sum-rate capacity for noisy interference}

The outer bound in Theorem 1 is in the form of an optimization problem. Four parameters $\rho_{1}, \rho_{2}, \sigma_{1}^{2}, \sigma_{2}^{2}$ need to be optimized for different choices of the weights $\mu, \eta_{1}, \eta_{2}$. When $\mu=1$, the bound (1) of Theorem 1 leads directly to the following sum-rate capacity result.

Theorem 2: For the $\operatorname{IC}\left(a, b, P_{1}, P_{2}\right)$ satisfying

$$
\sqrt{a}\left(b P_{1}+1\right)+\sqrt{b}\left(a P_{2}+1\right) \leq 1,
$$

the sum-rate capacity is

$$
C=\frac{1}{2} \log \left(1+\frac{P_{1}}{1+a P_{2}}\right)+\frac{1}{2} \log \left(1+\frac{P_{2}}{1+b P_{1}}\right) .
$$

Proof: By choosing

$$
\begin{aligned}
\sigma_{1}^{2}= & \frac{1}{2 b}\left\{b\left(a P_{2}+1\right)^{2}-a\left(b P_{1}+1\right)^{2}+1\right. \\
& \left. \pm \sqrt{\left[b\left(a P_{2}+1\right)^{2}-a\left(b P_{1}+1\right)^{2}+1\right]^{2}-4 b\left(a P_{2}+1\right)^{2}}\right\} \\
\sigma_{2}^{2}= & \frac{1}{2 a}\left\{a\left(b P_{1}+1\right)^{2}-b\left(a P_{2}+1\right)^{2}+1\right. \\
& \left. \pm \sqrt{\left[a\left(b P_{1}+1\right)^{2}-b\left(a P_{2}+1\right)^{2}+1\right]^{2}-4 a\left(b P_{1}+1\right)^{2}}\right\} \\
\rho_{1}= & \sqrt{1-a \sigma_{2}^{2}} \\
\rho_{2}= & \sqrt{1-b \sigma_{1}^{2}},
\end{aligned}
$$

the bound (1) with $\mu=1$ is

$$
R_{1}+R_{2} \leq \frac{1}{2} \log \left(1+\frac{P_{1}}{1+a P_{2}}\right)+\frac{1}{2} \log \left(1+\frac{P_{2}}{1+b P_{1}}\right) .
$$

But one can achieve equality in (19) by treating the interference as noise at both receivers. In order that the choices of $\sigma_{i}^{2}$ and $\rho_{i}^{2}$ are feasible, 15 must be satisfied.

Remark 7: Consider the bound (1) with $\mu=1$, we further let

$$
1-\rho_{1}^{2} \geq a \sigma_{2}^{2}, \quad 1-\rho_{2}^{2} \geq b \sigma_{1}^{2} .
$$

From (5) and (6) we have $P_{1}^{*}=P_{1}, P_{2}^{*}=P_{2}$. Thus,

$$
\begin{aligned}
R_{1} \leq & \frac{1}{2} \log \left(1+\frac{P_{1}}{\sigma_{1}^{2}}\right)-\frac{1}{2} \log \left(a P_{2}+1-\rho_{1}^{2}\right) \\
& +\frac{1}{2} \log \left[1+a P_{2}+P_{1}-\frac{\left(P_{1}+\rho_{1} \sigma_{1}\right)^{2}}{P_{1}+\sigma_{1}^{2}}\right] \\
= & \frac{1}{2} \log \left[\frac{P_{1}\left(1+a P_{2}\right)}{1+a P_{2}-\rho_{1}^{2}}\left(\frac{1}{\sigma_{1}}-\frac{\rho_{1}}{1+a P_{2}}\right)^{2}+1+\frac{P_{1}}{1+a P_{2}}\right] \\
\triangleq & f\left(\rho_{1}, \sigma_{1}\right) .
\end{aligned}
$$

Therefore, for any given $\rho_{1}$, when

$$
\rho_{1} \sigma_{1}=1+a P_{2},
$$

then $f\left(\rho_{1}, \sigma_{1}\right)$ achieves its minimum which is user 1 's singleuser detection rate. Similarly, we have $\rho_{2} \sigma_{2}=1+b P_{1}$. Since the constraint in (20) must be satisfied, we have

$$
\frac{1+a P_{2}}{\rho_{1}} \leq \sqrt{\frac{1-\rho_{2}^{2}}{b}}, \quad \frac{1+b P_{1}}{\rho_{2}} \leq \sqrt{\frac{1-\rho_{1}^{2}}{a}} .
$$

As long as there exists a $\rho_{i} \in(0,1)$ such that 23 is satisfied, we can choose $\sigma_{i}$ to satisfy (22) and hence the bound in (1) is tight. By cancelling $\rho_{1}, \rho_{2}$, we obtain 15 .

Remark 8: The most special choices of $\rho_{1}, \rho_{2}$ are in (17) and (18), since (11) and (12) with $\mu=1$ become

$$
\begin{aligned}
h\left(X_{1}^{n}+N_{1}^{n}\right)-h\left(\sqrt{b} X_{1}^{n}+Z_{2}^{n} \mid N_{2}^{n}\right) & =-n \log \sqrt{b} \\
h\left(X_{2}^{n}+N_{2}^{n}\right)-h\left(\sqrt{a} X_{2}^{n}+Z_{1}^{n} \mid N_{1}^{n}\right) & =-n \log \sqrt{a} .
\end{aligned}
$$


Therefore, we do not need the extremal inequality [17] to prove Theorem 2 .

Remark 9: The sum-rate capacity for a Z-IC with $a=0$, $0<b<1$ is a special case of Theorem 2 since (15) is satisfied. The sum-rate capacity is therefore given by (16).

Remark 10: Theorem 2 follows directly from Theorem 1 with $\mu=1$. It is remarkable that a genie-aided bound is tight if (15) is satisfied since the genie provides extra signals to the receivers without increasing the rates. This situation is reminiscent of the recent capacity results for vector Gaussian broadcast channels (see [19]). Furthermore, the sumrate capacity (16) is achieved by treating the interference as noise. We therefore refer to channels satisfying (15) as ICs with noisy interference.

Remark 11: For a symmetric IC where $a=b, P_{1}=P_{2}=P$, the constraint (15) implies that

$$
a \leq \frac{1}{4}, \quad P \leq \frac{\sqrt{a}-2 a}{2 a^{2}} .
$$

Noisy interference is therefore weaker than weak interference as defined in [9] and [20], namely $a \leq \frac{\sqrt{1+2 P}-1}{2 P}$ or

$$
a \leq \frac{1}{2}, \quad P \leq \frac{1-2 a}{2 a^{2}} .
$$

Recall that [20] showed that for "weak interference" satisfying (25), treating interference as noise achieves larger sum rate than time- or frequency-division multiplexing (TDM/FDM), and [9] claimed that in "weak interference" the largest known achievable sum rate is achieved by treating the interference as noise.

\section{Capacity region corner point}

The bounds (2) and (3) of Theorem 1 lead to the following sum-rate capacity result.

Theorem 3: For an $\operatorname{IC}\left(a, b, P_{1}, P_{2}\right)$ with $a>1,0<b<1$, the sum-rate capacity is

$$
C=\frac{1}{2} \log \left(1+P_{1}\right)+\frac{1}{2} \log \left(1+\frac{P_{2}}{1+b P_{1}}\right)
$$

when the following condition holds

$$
(1-a b) P_{1} \leq a-1 .
$$

A similar result follows by swapping $a$ and $b$, and $P_{1}$ and $P_{2}$.

This sum-rate capacity is achieved by a simple scheme: user 1 transmits at the maximum rate and user 2 transmits at the rate that both receivers can decode its message with singleuser detection. Such a rate constraint was considered in [9, Theorem 1] which established a corner point of the capacity region. However it was pointed out in [20] that the proof in [9] was flawed. Theorem 3 shows that the rate pair of [20] is in fact a corner point of the capacity region when $a>1,0<$ $b<1$ and (27) is satisfied, and this rate pair achieves the sum-rate capacity.

The sum-rate capacity of the degraded IC $(a b=1,0<b<$ 1 ) is a special case of Theorem 3 . Besides this example, there are two other kinds of ICs to which Theorem 3 applies. The first case is $a b>1$. In this case, $P_{1}$ can be any positive value. The second case is $a b<1$ and $P_{1} \leq \frac{a-1}{1-a b}$. For both cases, the signals from user 2 can be decoded first at both receivers.

\section{SUM-RATE CAPACITY FOR $m$-USER IC WITH NOISY INTERFERENCE}

For an $m$-user IC, the receive signal at user $i$ is defined as

$$
Y_{i}=X_{i}+\sum_{j=1, j \neq i}^{m}\left(\sqrt{c_{j i}} X_{j}\right)+Z_{i}, \quad i=1,2, \ldots, m,
$$

where $c_{j i}$ is the channel gain from $j^{t h}$ transmitter to $i^{t h}$ receiver, $Z_{i}$ is unit-variance Gaussian noise, and the transmit signals have the block power constraints $\sum_{l=1}^{n} \mathcal{E}\left(X_{i l}^{2}\right) \leq n P_{i}$. We have the following sum-rate capacity result.

Theorem 4: For an $m$-user IC defined in (28), if there exist $\rho_{i} \in(0,1), i=1, \ldots, m$, such that the following conditions are satisfied

$$
\begin{aligned}
\sum_{j=1, j \neq i}^{m} \frac{c_{j i}\left(1+Q_{j}\right)^{2}}{\rho_{j}^{2}} & \leq 1-\rho_{i}^{2} \\
\sum_{j=1, j \neq i}^{m} \frac{c_{i j}}{1+Q_{j}-\rho_{j}^{2}} & \leq \frac{1}{P_{i}+\left(1+Q_{i}\right)^{2} / \rho_{i}^{2}},
\end{aligned}
$$

where $Q_{i}$ is the interference power at receiver $i$, defined as

$$
Q_{i}=\sum_{j=1, j \neq i}^{m} c_{j i} P_{j},
$$

the sum-rate capacity is

$$
C=\frac{1}{2} \sum_{i=1}^{m} \log \left(1+\frac{P_{i}}{1+Q_{i}}\right)
$$

Therefore, if there exist $\rho_{1}, \ldots, \rho_{m}$, such that (29) and (30) are satisfied for all $i=1, \ldots, m$, the sum-rate capacity of an $m$-user IC can be achieved by treating interference as noise. The proof is omitted due to the space limitation. It can be shown that Theorem 2 is a special case of Theorem 4

Consider a uniformly symmetric $m$-user IC where $c_{j i}=c$, for all $i, j=1, \ldots, m, i \neq j$, and $P_{i}=P$. The bounds 29) and (30) with $\rho_{i}=\rho$ for all $i$ reduce to

$$
c \leq \frac{1}{4(m-1)}, \quad P \leq \frac{\sqrt{(m-1) c}-2(m-1) c}{2(m-1)^{2} c^{2}} .
$$

\section{NUMERICAL EXAMPLES}

A comparison of the outer bounds for a Gaussian IC is given in Fig. 11 Some part of the outer bound from Theorem (1) overlaps with Kramer's outer bound due to (2) and (3). Since this IC has noisy interference, the proposed outer bound coincides with the inner bound at the sum rate point.

The lower and upper bounds for the sum-rate capacity of the symmetric IC are shown in Fig 2 for high power level. The upper bound is tight up to point $A$. The bound in [15, Theorem 3] approaches the bound in Theorem 1 when the power is large, but there is still a gap. Fig. 2 also provides a definitive answer to a question from [20, Fig. 2]: whether the 
sum-rate capacity of symmetric Gaussian IC is a decreasing function of $a$, or there exists a bump like the lower bound when $a$ varies from 0 to 1. In Fig. 2, our proposed upper bound and Sason's inner bound explicitly show that the sum capacity is not a monotone function of $a$ (this result also follows by the bounds of [15]).

\section{CONCLUSIONS, EXTENSIONS AND PARALLEL WORK}

We derived an outer bound for the capacity region of 2user Gaussian ICs by a genie-aided method. From this outer bound, the sum-rate capacities for ICs that satisfy (15) or 27) are obtained. The sum-rate capacity for $m$-user Gaussian ICs that satisfy (29) and (30) are also obtained.

Finally, we wish to acknowledge parallel work. After submitting our 2-user bounds and capacity results on the arXiv.org e-Print archive [2], two other teams of researchers - Motahari and Khandani from the University of Waterloo, Annapureddy and Veeravalli from the University of Illinois at UrbanaChampaign - let us know that they derived the same 2-user sum-rate capacity results (Theorem 2).

\section{Acknowledgement}

The work of X. Shang and B. Chen was supported in part by the NSF under Grants 0546491 and 0501534 , and by the AFOSR under Grant FA9550-06-1-0051, and by the AFRL under Agreement FA8750-05-2-0121.

G. Kramer gratefully acknowledges the support of the Board of Trustees of the University of Illinois Subaward no. 04-217 under NSF Grant CCR-0325673 and the Army Research Office under ARO Grant W911NF-06-1-0182.

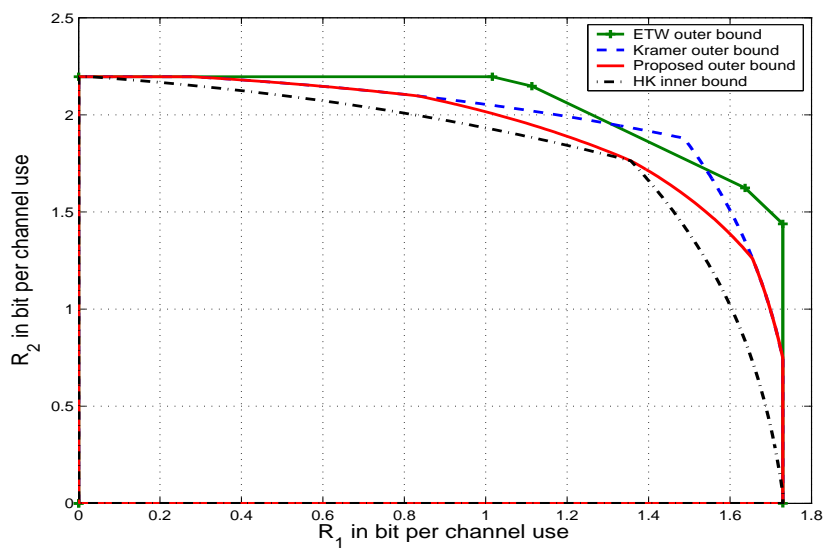

Fig. 1. Inner and outer bounds for the capacity region of Gaussian ICs with $a=0.04, b=0.09, P_{1}=10, P_{2}=20$. The ETW bound is by Etkin, Tse and Wang in [15, Theorem 3]; the Kramer bound is from [14, Theorem 2]; the HK inner bound is based on [8] by Han and Kobayashi.

\section{REFERENCES}

[1] X. Shang, G. Kramer, and B. Chen, "Outer bound and noisy-interference sum-rate capacity for symmetric Gaussian interference channels," submitted to CISS 2008

[2] X. Shang, G. Kramer, and B. Chen, "A new outer bound and the noisy-interference sum-rate capacity for Gaussian interference channels," submitted to the IEEE Trans. Inform. Theory. http://arxiv.org/abs/0712.1987, 2007.

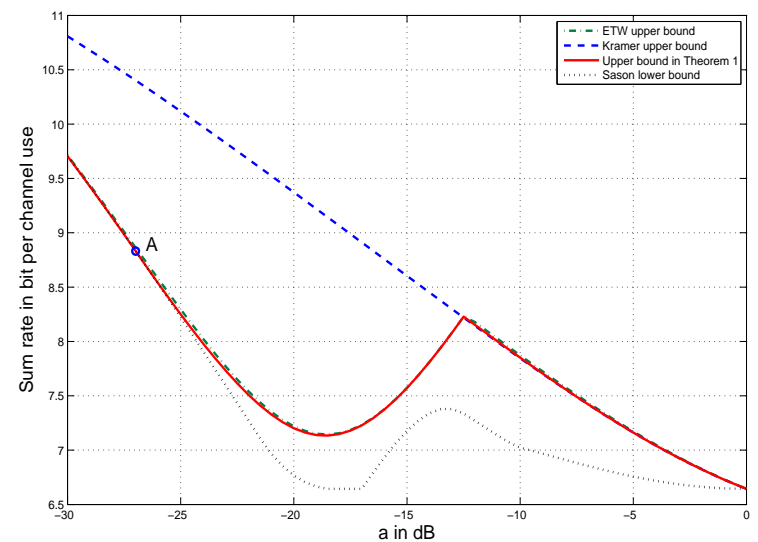

Fig. 2. Lower and upper bounds for the sum-rate capacity of symmetric Gaussian ICs with $a=b, P_{1}=P_{2}=5000$. The channel gain at point $A$ is $a=-26.99 \mathrm{~dB}$. Sason's bound is an inner bound obtained from Han and Kobayashi's bound by a special time sharing scheme [20, Table I]

[3] A. S. Motahari and A. K. Khandani, "Capacity bounds for the Gaussian interference channel," to be submitted to IEEE Trans. Inform. Theory.

[4] V. S. Annapureddy and V. Veeravalli, "Sum capacity of the Gaussian interference channel in the low interference regime," submitted to ITA 2008.

[5] A.B. Carleial, "Interference Channels," IEEE Trans. Inform. Theory, vol. 24, pp. 60-70, Jan. 1978.

[6] A.B. Carleial, "A case where interference does not reduce capacity," IEEE Trans. Inform. Theory, vol. 21, pp. 569-570, Sep. 1975.

[7] H. Sato, "The capacity of the Gaussian interference channel under strong interference," IEEE Trans. Inform. Theory, vol. 27, pp. 786-788, Nov. 1981.

[8] T.S. Han and K. Kobayashi, "A new achievable rate region for the interference channel," IEEE Trans. Inform. Theory, vol. 27, pp. 49-60, Jan. 1981.

[9] M.H.M. Costa, "On the Gaussian interference channel," IEEE Trans. Inform. Theory, vol. 31, pp. 607-615, Sept. 1985.

[10] H.F. Chong, M. Motani, H.K. Garg, and H.E. Gamal, "On the HanKobayashi Region for the interference channel," submitted to the IEEE Trans. Inform. Theory, 2006.

[11] G. Kramer, "Review of rate regions for interference channels," in International Zurich Seminar, Zurich, Feb.22-24 2006, pp. 162-165.

[12] H. Sato, "Two-user communication channels," IEEE Trans. Inform. Theory, vol. 23, pp. 295-304, May 1977.

[13] A.B. Carleial, "Outer bounds on the capacity of interference channels," IEEE Trans. Inform. Theory, vol. 29, pp. 602-606, July 1983.

[14] G. Kramer, "Outer bounds on the capacity of Gaussian interference channels," IEEE Trans. on Inform. Theory, vol. 50, pp. 581-586, Mar. 2004.

[15] R. H. Etkin, D. N. C. Tse, and H. Wang, "Gaussian interference channel capacity to within one bit," submitted to the IEEE Trans. Inform. Theory, 2007.

[16] E. Telatar and D. Tse, "Bounds on the capacity region of a class of interference channels," in Proc. IEEE International Symposium on Information Theory 2007, Nice, France, Jun. 2007.

[17] T. Liu and P. Viswanath, "An extremal inequality motivated by multiterminal information-theoretic problems," IEEE Trans. Inform. Theory, vol. 53, no. 5, pp. 1839-1851, May 2006

[18] H. Sato, "On degraded Gaussian two-user channels," IEEE Trans. Inform. Theory, vol. 24, pp. 634-640, Sept. 1978.

[19] H. Weingarten, Y. Steinberg, and S. Shamai (Shitz), "The capacity region of the Gaussian multiple-input multiple-output broadcast channel," IEEE Trans. Inform. Theory, vol. 52, no. 9, pp. 3936-3964, Sep. 2006.

[20] I. Sason, "On achievable rate regions for the Gaussian interference channels," IEEE Trans. Inform. Theory, vol. 50, pp. 1345-1356, June 2004. 